\title{
Investigation in Effect of Nano-WC powder on wear resistance of Fe/ WC coating
}

\author{
Hongjian Guo ${ }^{1 a}$, Zhenyu Zhang $^{1 \mathrm{~b}}$, Bunv Liang ${ }^{1 \mathrm{c}}$, Jing Li ${ }^{1 \mathrm{~d}}$, Xiaojun Lin $^{1 \mathrm{e}}$ \\ 1.Department of Material Engineering ,Lanzhou Institute of Technology, LanZhou, 730050 China

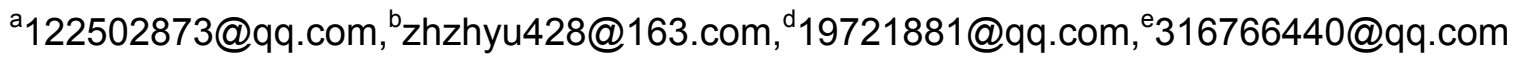

Keywords: Subsonic flame spraying; Nano-WC;Fe/WC coating;wear resistance

\begin{abstract}
In view of the wear and corrosion damage of water turbine in the hydropower station, the different content weight rations of nano-WC powders were added into traditional metal--ceramic coating materials Fe/ WC, the composite coatings were manufactured on the surface of $45 \#$ steel by the Subsonic flame spraying, the microstructure and properties of coatings were studied, the influence of the nano-WC powders on the composite coating were researched.The result showed that, with suitable addition of the nano-WC in the Fe/WC coating, the microstructure of the spraying coating could be refined, microhardness and wear resistance of the coating could be increased.
\end{abstract}

\section{Introduction}

By virtue of their unique advantages in performance, ceramic materials are widely used in thermal spray coatings, the wear-resistant, corrosion-resistant ceramic coating can be obtained. In recent years, the papers about nano-modification ceramic coatings and nano-structured ceramic coatings are[1,2] more and more, they have become the hot research topics focus on the thermal spray technology. The literature reported[3], on the surface of ordinary substrate ,the integrity of sprayed WC coating was good, and wear and corrosion resistance performance was 4.6 times more than that of the 18-8 stainless steel, but the reportes about the nano-WC was added into the spraying materials as additives were rare. In this article, different contents of nano-WC was added into the traditional spraying materials Fe/WC ,Effect of nano-WC powder on wear resistance of thermal spraying coating was studyed, and found the best dosage.

\section{Experimental Procedures}

Sample preparation. The substrate of the sample was $45 \#$ steel, sizes were $\Phi 20 \mathrm{~mm} \times 5 \mathrm{~mm}$ (for Microstructure test, microhardness test, XRD test) and $\Phi 20 \mathrm{~mm} \times 18 \mathrm{~mm}$ (for wear resistance test). A small amount of nano-Ni, nano- $\mathrm{CeO}_{2}$ and different contents of nano-WC are added into $\mathrm{Fe}-\mathrm{WC}$ spray materials, The spraying powders are mixed mechanically in a dry state. Addition proportion of nano-Ni $(50 \sim 70 \mathrm{~nm})$ was wt. $\%$, addition proportion of nano-WC( $20 \sim 50 \mathrm{~nm})$ were $0 \%$, wt. $2 \%$, wt. $4 \%$, wt. $6 \%$, addition proportion of nano- $\mathrm{CeO}_{2}(20 \sim 60 \mathrm{~nm})$ was wt. $1 \%$, addition proportion of micron-WC(250 300 mesh) was 30\% and the margin was Fe(200 300 mesh) .

Experimental methods. The ceramic coating was obtained by subsonic flame spraying method, the parameters were kept unchanged constantly, Oxygen pressure was $0.8 \mathrm{Mpa}$, Ethine pressure was1.1 1.2Mpa, Air pressure was 0.4 Mpa, Distance of spraying was $200 \sim 300 \mathrm{~mm}$, angle of spraying $45^{\circ} \sim 90^{\circ}$, thickness of coating $04 \sim 0.6 \mathrm{~mm}$. Friction and wear tests were conduced on MM-W1 wear tester, Grinding materials was 180 \# abrasive paper, the tests were carried out out at sliding speed of $200 \mathrm{rpm} / \mathrm{min}$, sliding load of $60 \mathrm{~N}$, and sliding time of $10 \mathrm{~min}$. the surface morphology of coatings were conduced on SN-3400 scanning electron microscope(made by Hitachi company, Japan), microhardness were conduced on HXD-1000TM microhardness meter test, the tests were carried out at load of $200 \mathrm{~kg}$,load time of 15 seconds each,respectively, and the phases of composites were characterized by XRD.

\section{Results and Discussion}


Microstructure.Fig. 1 shows cross-section microstructure of spraying coating. According to the results of previous researchers [4,5], the molten metal ceramic powders hit the surface of substrate at the high-speed in the air flow, we can find the typical lamellar morphology in the figure, black oxides organizations formed during spraying between the lamellar structure, and a small amount of pores in the coating. It can be seen that the grains of the coating without nano-WC combine poorly, the microstructure of the coating is relatively porosity, mixed with a certain amount of holes (shown in Figure 1a I ) and the crack, which greatly reduces the bonding strength and abrasion resistance; It also can be seen that the microstructure of the coating added only $2 \%$ nano-WC are more compact and uniform than the coating without nano-WC coating, and the pores in the coating are few, the combination of coating and substrate is relatively fine; morphology of the coating add 4\% nano-WC is the best, coating grains are obviously refined, almost no holes in the coating, compactness of the coating is also fine, the coating combines the substrate closely; but the microstructure of the coating with the addition of $6 \%$ nano-WC are coarse, and mixed with some pores, seemingly exists internal grain accumulation and reunion, as shown in Figure 1d II.

In summary, with the different addition of nano-WC, the microstucture of the coating change significantly, it can be summarized that nano-WC affected the microstructure of the Fe/WC coating layer, when the coating with the addition of $4 \%$ nano-WC, it can be obviously refined,with the best combination between the dense coating and the substrate .
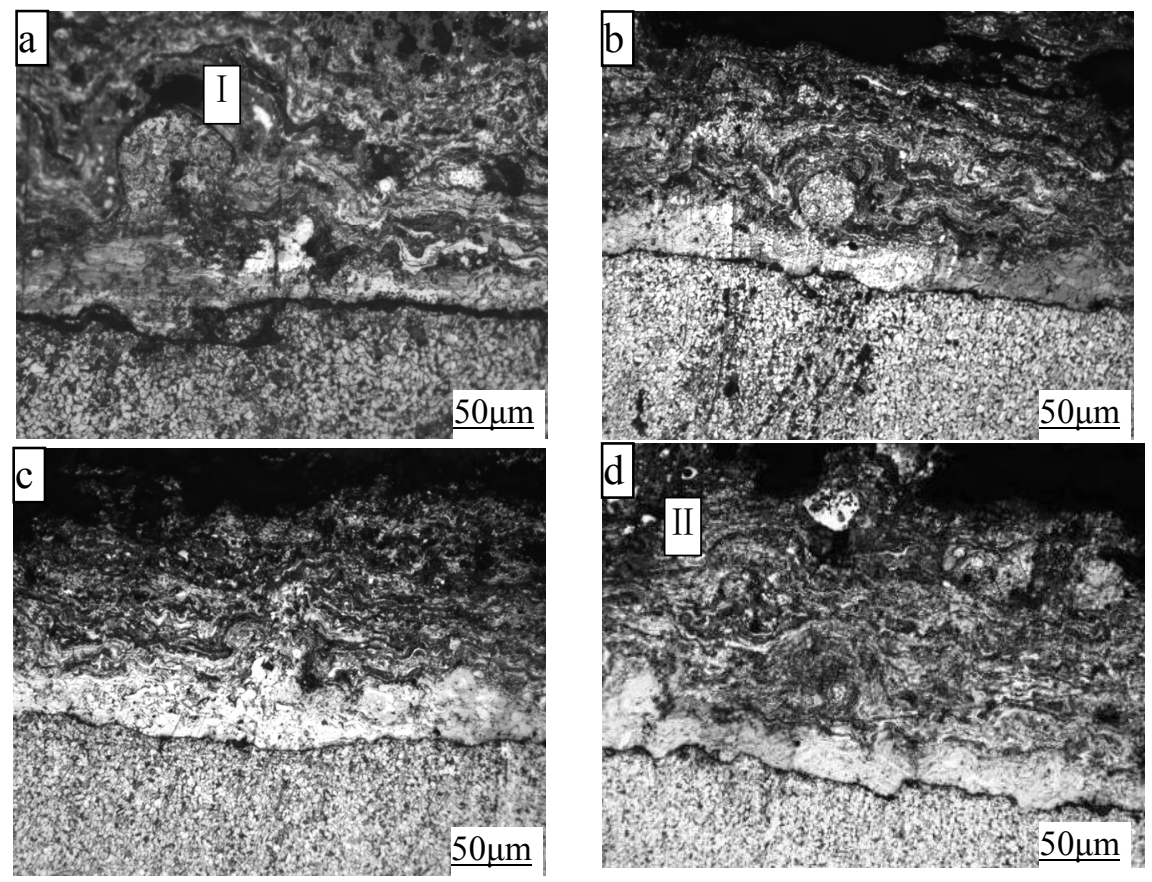

Fig.1Microstructure of coatings with the different amount of nano- WC: (a) $0 \%$ nano-WC

(b) $2 \%$ nano-WC (c) $4 \%$ nano-WC (d) $6 \%$ nano-WC

Through a series of analysis, it can be seen that small nano-WC particles have large surface area and high activity, increase the degree of weting among the particles in the coating, promote the combination of the coating particles, furthermore , melting performance of nano-WC is good[6], in large touching deformation to the substrate surface, the combination of the coating is tight; the compactness is increased.The nano-WC particles appear Liquid phase sintering[7] in the cooling process, the nano-WC always congeales later than conventional micron particles. And coupled with the surface effect of nanoparticles, with high chemical activity, the nano-WC can easily combine with other atoms to form stable compounds within the coatings, particles appear micro-metallurgical bond, and reduce the Oxygen、Sulfur and other non-metallic element content, and resulting in less stoma ,to enhance the compactness of the coating.

The phases of the coating with $4 \%$ nano-WC were tested on X-ray diffractometer,the result shows that the $\mathrm{WC}$ is decomposed into $\mathrm{W}_{2} \mathrm{C}$ and $\mathrm{W}$ in the high-temperature oxygen - acetylene flame. With the increasing addition of nano-WC, the amount of free carbon content is more and more in the 
composite coating, with the decomposition of WC [8], free carbon is oxidized into carbon dioxide gas which can not be completely released from the coating,leaving a large number of pores, which damages the compactness and the metallurgical bonding of the coating, consequently, mixed with the coarse tissue and the pores in the coating with $6 \%$ nano-WC.

\section{microhardness.}

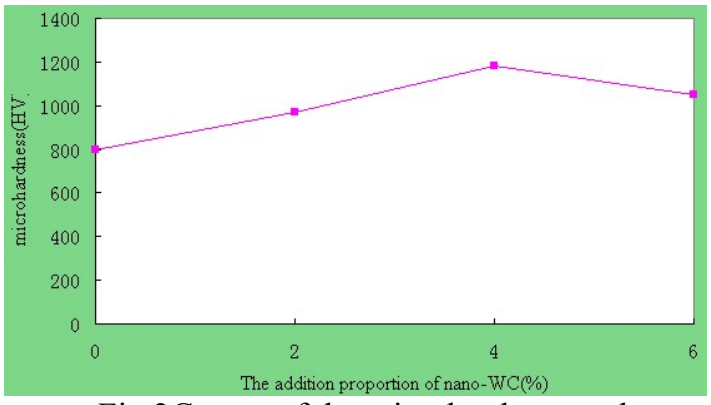

Fig.2Curves of the microhardness and the addition proportion of nano-WC

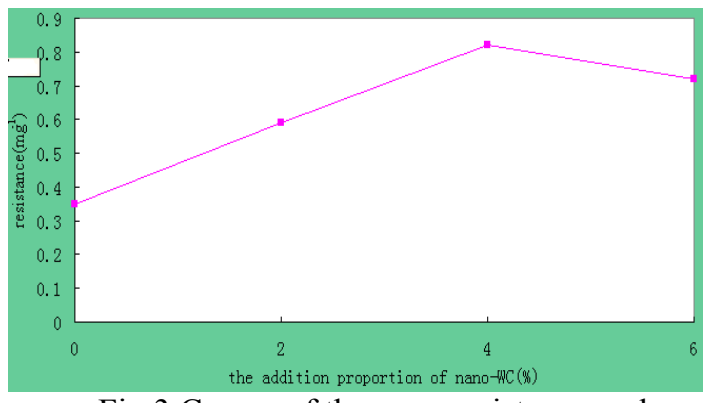

Fig. 3 Curves of the wear resistance and the addition proportion of nano-WC

Fig.2 shows relationship between the microhardness and the addition proportion of nano-WC.It can be seen, with the increase of nano-WC, the microhardness of the coating shows the trend of increase firstly and then reduce.

After a serious of analysis,there may be some reasons as following.Firstly, nano-WC particles playe the role of dispersion strengthening in the coating [9], so as to improve the microhardness; secondly, the nano-WC is very hard, its microhardness is up to HV2000 , under the synthesized effects of dispersion strengthening and steady by the nano-WC and micron-WC, microhardness of the coating also increased accordingly. Because the density of the $\mathrm{WC}$ is very high,when the addition amount of $\mathrm{WC}$ is up to $6 \%$, due to the gravity segregation and agglomeration of $\mathrm{WC}$ particles, resulting in uneven distribution of coating composition, and performance of the coating as a whole is uneven too; As mentioned above, due to the decarburization decomposition of $\mathrm{WC}$, resulting in the increase of microscopic holes in the coating, resulting in the porosity of tissue in the coating, resulting in the debasement of toughness of the coating, ultimately resulting in debasement of microhardness [10].

\section{Wear Resistance.}
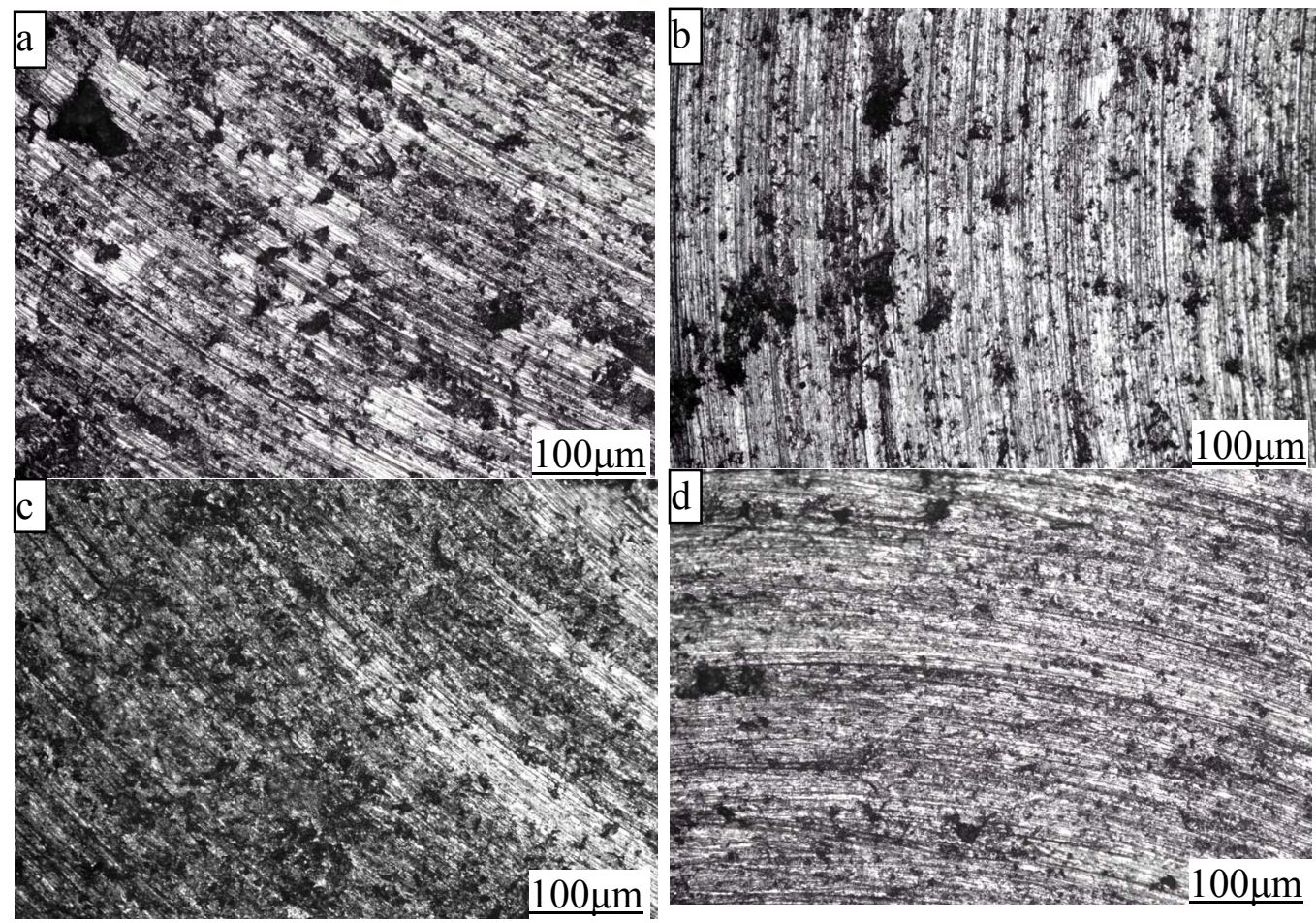

Fig.4 Surface appearance of the coatings after wear:（a）0\%nano-WC（b）2\% nano-WC（c） 4\% nano-WC（a）6\% nano-WC 
Fig. 3 shows the relationship between the addition amount of Nano-WC and wear resistance. It can be seen from the figure, with the increase of nano-WC, the wear resistance of the coating shows the trend of increase firstly and then reduce, the wear resistance of the coating with $4 \%$ nano-WC is the best.

Fig. 4 shows the surface appearance of the coating after wear, according to the results of resistance test, it can be seen that the lower the wear resistance, the more serious the coating peeling off , and there is a very obvious difference between the coating added nano-WC and not added. On the surface of the coating with Nano-WC powders, due to exfoliation of the hard phase WC, there are a large number of black pits on the surface of the coating, there are clear scratches, that wide and deep. Compared with the coating without nano-WC, surface of the coating with nano-WC is relatively smooth, exfoliation of the coating is relatively less. But the degree of abrasion of the coatings is diferent because of the different addition of nano-WC, compared with the wear surface of the coatings with nano-WC $2 \%, 4 \% 、 6 \%$,furthermore, it can be seen that there are also some pits and nick on surface of the coating with $2 \%$ nano-WC after wear, but relatively shallow scratches. Compared with the coating with nano-WC $4 \%, 6 \%$, there is no obvious scratches and exfoliation on the wear surface, there is no exfoliation almost on the surface of the coating with $4 \%$ nano-WC after wear, and only a small amount of very shallow plastic plow deformation. The degree of abrasion of the coating with nano-WC6\% is more serious than that of the coating with nano-WC $4 \%$.In conclusion, Nano-WC can improve the wear resistance of the coating due to its special properties.

SEM, XRD tests of the coatings show that,due to "Nano effect" of nano-WC particles,resulting in grain refinement of the composite coating, dispersion strengthening of the superhard particles in the coatings, ultimatly, resulting in high wear resistance of the coating. When the addition proportion of nano-WC is $6 \%$, tissue of the spraying coating changes into loose, the toughness of the coating decreases, the wear resistance decreases finaly,it may be result of that the addition proportion of nano-WC is too much .

\section{Conclusions}

(1) The nano-WC can improve the microstructure of the coating if the addition proportion is suitable, the best addition proportion of nano-WC is $4 \%$.

(2) The nano-WC can increase the microhardness and wear resistance of the coating. When the addition proportion of nano-WC is $4 \%$, the microhardness and wear resistance is the best.

\section{Acknowledgements}

The project was supported by the Gansu Province Natural Science Foundation(1107RJZ119).

The authors are grateful to State Key Laboratory of Solid Lubrication, Lanzhou Institute of Chemical Physics, Chinese Academy of Sciences for providing experimental devices.Professor ZhangZhen-yu is highly appreciated for his assistance in English writing.

\section{References}

[1] Y.H.Guan, Y.Xu, Z.Y.Zheng:Tribology, Vol.26(2006),p.320

[2] Y.G.Yan, M.X.Li, S.H Zhang: Journal of the Chinese Rare Earth Society, Vol.25(2007),P.620

[3] G.Deng,Y.P.Song, W.Y.Wang:Hot Working Technology, Vol. 5( 2005),p.14

[4] C.Z Chen, H.J.Yu, W.Z.Wang: Journal of Ceramic,Vol.20(1999),P.141

[5] D.Y.He, B.Y.Fu, J.M.Jiang: Tribology,Vol .27(2007),p.116

[6] X.Q.Zhao,H.D. Zhou, J.M.Chen: Tribology,Vol .26(2006),P.208

[7] C.J. Qiu, R.L.Liu, X.F.Fan: Chinese Journal of Material Research, Vol.15(2001),P.479

[8] C.Q.Sun:Research on the Characteristics of Low-power Plasma Spraying Tungsten Carbide

[D].Da Lian, Dalian Maritime University(2005)

[9] D.Y.He,F.Y.Zhang,J.M.Jiang: China Surface Engineering, Vol.19(2006),P.41

[10] Y.Gao, Y.Q.Shi, G.Xin: Materials For Mechanical Engineering, Vol.26(2006),p.25 